\title{
Blind Known Interference Cancellation with Parallel Real Valued Belief Propagation Algorithm
}

\author{
Shengli Zhang*, Soung Chang Liew ${ }^{\S}$ Lu Lu ${ }^{\S}$ Hui Wang* \\ *MCSP key lab of Shenzhen, Communication Engineering Department, Shenzhen University, Shenzhen, China \\ ${ }^{\S}$ Information Engineering Department, Chinese University of Hong Kong, Hong Kong, China
}

\begin{abstract}
This paper investigates interference-cancellation schemes at the receiver, in which the original data of the interference is known a priori. Such a priori knowledge is common in wireless relay networks. Directly removing the known interference requires accurate estimate of the interference channel, which may be difficult in many situations. In [1], we proposed a novel scheme, Blind Known Interference Cancellation (BKIC), for blind cancellation of known interference without interference channel information. BKIC consists of two steps. The first step combines adjacent symbols to cancel the interference, exploiting the fact that the channel coefficients are almost the same between successive symbols. After such interference cancellation, however, the signal of interest is also distorted. The second step recovers the signal of interest amidst the distortion. Two schemes for the second step, BKIC-S and successive BKIC-RBP, were proposed in [1]. BKIC-S removes distortion by smoothing while BKIC-RBP does so using a real-value belief propagation algorithm. Although successive BKIC-RBP performs well and is superior to BKIC-S, it requires a long processing time proportional to the packet length. To overcome this problem, this paper proposes a parallel BKIC-RBP algorithm. Parallel BKIC-RBP has similar performance as successive BKIC-RBP. It has the advantage of being amenable to parallel implementation with a much shorter processing time.
\end{abstract}

\section{Introduction}

In wireless relay networks, a node may receive a target signal superimposed with interferences. However, in many scenarios, the receiver actually knows the data contained in the interference [2], either because the interference was signal previously received by the node, or the interference is self-information previously transmitted by the node and now transmitted by the relay superimposed with signal from another node. For example, when physical-layer network coding over infinite field $[3,15,16]$ (analog network coding [4]) is used in a two-way relay channel, as shown in Fig. 1, the signal transmitted by the relay is a superposition of the signals from the two end nodes; as far as the reception at an end node is concerned, the "interference" within the superimposed signal is actually self-information.

Conventional method for dealing with known interference is straightforward in theory. The receiver first estimates the channel coefficient associated with the interference signal and then removes the known interference from the received signal [2]. We refer to this scheme as traditional KIC (Known Interference Cancellation) throughout this paper. In practice, however, the scheme does not perform well when channel estimation is inaccurate.

Accurate channel estimation is a non-trivial problem even in the absence of interference. In the presence of interference, we face additional challenges because both the channels of the targeted signal and interference must be estimated. There has been some work trying to tackle this problem. To estimate two channels simultaneously, the physical-layer network coding implementation in [5] uses orthogonal sequences for the two packets. The analog network coding scheme in [4], on the other hand, attaches the training sequence to both the front end and back end of a packet, and time the transmissions of the two packets so that one of them has interference-free front end and the other one has interference-free back end. These schemes use new frame designs and are not compatible with legacy wireless systems. Ref. [6] uses an optimization scheme to estimate the channels with two specially designed training sequences. The estimation accuracy is much poorer than that in single-channel estimation. Non-coherent ANC schemes that avoid channel estimation altogether have also been studied [7, 8]. However, non-coherent schemes suffer from SNR degradation of about $3 \mathrm{~dB}$ compared with the coherent schemes. By contrast, the blind known interference cancellation (BKIC) schemes considered in this paper can obtain near-perfect performance - specifically, performance close to that of a point-to-point communication link without interference - while avoiding estimation of the interference channel.

We first proposed the BKIC scheme in [1]. In general, BKIC has three advantages over the traditional methods: 1) better performance (very close to the upper bound); 2) no need for interference channel estimation; and 3) lower complexity and compatibility with legacy systems. BKIC operates on the principle that wireless channels typically remain almost unchanged between adjacent symbols [9]. BKIC uses the interference in one symbol to cancel the interference in its adjacent symbol. For example, if the interference channel is $h$ and the interference symbol is 1 , then the interference in the current symbol is $h$. If the interference data in the adjacent symbol is -1 , then the corresponding known interference is approximately $-h$. The interference can be cancelled with each other if we combine the two symbols. Such adjacent-symbol combination, however, may result in distortion of the target signal. Thus, a key issue is how to remove such distortion as the next step. Two schemes, smoothing (BKIC-S) and real-valued belief propagation (BKIC-RBP), were proposed in [1] to equalize the resulting distortion.

It was shown in [1] BKIC-RBP scheme performs much better than the BKIC-S scheme, especially in continuous fading channel. In this paper, we first point out that BKIC-S is essentially the traditional KIC with correlation based channel 
estimation. This means that BKIC-RBP has performance superior to correlation-based KIC. After that, we propose a parallel BKIC-RBP scheme. Compared to the successive BKIC-RBP in [1], the new parallel scheme can significantly reduce the processing time. Simulation shows that the new parallel BKIC-RBP scheme has similar performance as the successive BKIC-RBP in [1].

The remainder of this paper is organized as follows. In section II, we present the system model and notations of BKIC. Section III reviews basic BKIC scheme. We propose the parallel BKIC-RBP scheme in Section IV. The performance of the new scheme is validated in Section $\mathrm{V}$ by simulation. Finally, Section VI concludes this paper.

\section{System Model and Architecture}

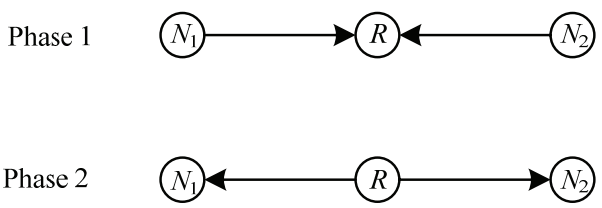

Fig. 1. Two way relay with analog network coding

Consider the two way relay channel in Fig. 1 where the two end nodes exchange information through the relay node. The transmission cycle consists of two phases. In the first phase, the two end nodes transmit their packets to the relay simultaneously. We assume the packet arrives at the relay with symbol level synchronization. Thus, the relay receives

$$
r(k)=h_{1, r}(k) x_{1}(k)+h_{2, r}(k) x_{2}(k)+n(k)
$$

where $x_{i}(k)$ is the $k$-th symbol of node $N_{i}$ and $h_{i, r}(k)$ is the corresponding channel coefficient from node $N_{i}$ to $R$. After receiving the signal, the relay node amplifies and broadcasts it to both end nodes. The received signal at $N_{1}$ is

$$
\begin{aligned}
& y(k)=\beta h_{r, 1}(k)\left[h_{1, r}(k) x_{1}(k)+h_{2, r}(k) x_{2}(k)+n_{r}(k)\right]+n_{1}(k) \\
& =\sqrt{P_{2}} g_{2}(k) x_{2}(k)+\sqrt{P_{1}} g_{1}(k) x_{1}(k)+n(k)
\end{aligned}
$$

where $g_{1}(k)$ and $g_{2}(k)$ are the equivalent channel coefficients seen by $N_{1}$ and $n(k)$ is the equivalent Gaussian noise. In this paper, the equivalent channel is assumed to incur flat continuous fading that varies with time with the first-order Markov channel mode [10]:

$$
g_{i}(k)=\sqrt{1-\alpha^{2}} g_{i}(k-1)+\alpha v
$$

where $g_{i}(k)$ and $v$ are Gaussian random variables with distribution $N(0,1)$, and $\alpha$ is the coefficient that characterizes the speed at which the channel changes.

In (2), $N_{1}$ knows $x_{1}(k)$, and it transforms $y(k)$ to the desired signal plus noise (DSN), $\sqrt{P_{2}} g_{2}(k) x_{2}(k)+n(k)$, plus a small interference as

$$
\begin{aligned}
& z(k)=\sqrt{P_{2}} g_{2}(k) x_{2}(k)+n(k)+w(k) \\
& =x^{\prime}(k)+n(k)+w(k)
\end{aligned}
$$

before detecting $x_{2}(k)$. In (4), $w(k)$ is the residual interference after known interference cancellation.

\section{Review of BKIC}

In this section, we review the BKIC scheme in [1]. Before that, the Traditional KIC scheme is given to show its equivalent to BKIC-S.

\section{III.1 Traditional KIC Scheme:}

In slow fading channel, one well known conventional scheme first estimates the interference channel coefficient by taking correlation with the known interference signal as

$$
\begin{aligned}
& g_{1, e}=\sum_{k=1}^{N} y(k) x_{1}(k) / \sqrt{P}_{1} \sum_{k=1}^{N}\left|x_{1}(k)\right|^{2} \\
& =\frac{1}{N} \sum_{k=1}^{N} g_{1}(k)+\frac{\sqrt{P_{2}}}{N \sqrt{P_{1}}} \sum_{k=1}^{N} g_{2} x_{2}(k) x_{1}(k)+\frac{1}{N} \sum_{k=1}^{N} n(k) x_{1}(k)
\end{aligned}
$$

where $N$ is the packet length. When $g_{1}(k)$ is constant within the packet, the first item in (5) is simply the constant $g_{1}(k)$. In practice, the channel is always time varying, and the variation depends on the moving speed of the terminal and other environmental factors. However, for adjacent symbols, the channel variation is very small. Then we can approximate the channel variation as

$$
g_{1}(k+1) \approx g_{1}(k)+\Delta(k) .
$$

The estimation error can be further simplified as

$$
\begin{aligned}
g_{1}(m)-g_{1, e} & \approx \sum_{k=m+1}^{N}(N-k) \Delta(k-1)-\sum_{k=1}^{m-1} k \Delta(k) \\
& +\frac{\sqrt{P_{2}}}{N \sqrt{P_{1}}} \sum_{k=1}^{N} g_{2} x_{2}(k) x_{1}(k)+\frac{1}{N} \sum_{k=1}^{N} n(k) x_{1}(k)
\end{aligned}
$$

From (7), we can see that a larger $N$ will decrease the residual interference and noise (the last two terms in (7)) and will increase the estimation error (the first two terms in (7)). Then the DSN can be estimated by removing the interference as

$$
z(k)=x^{\prime}(k)+\sqrt{P_{1}} x_{1}(k)\left(g_{1}(k)-g_{1}\right)+n(k)
$$

This equation is actually equivalent to Eq. (8) in [1], which shows the equivalence of correlation-based KIC to the BKIC-S.

\section{2 BKIC Scheme:}

BKIC consists of two steps. In the first step, the interference is canceled by combining adjacent symbols. In the second step, the DSN (distorted during the cancellation step), is recovered. The second step is the non-trivial step, and a Belief Propagation algorithm was proposed for it. 


\section{Step 1: Interference Cancellation}

In (6), $\Delta(k)$ is governed by the Doppler rate, and it is almost negligible in modern wireless communication systems $[11,12]$. This is a key observation in this paper that enables us to use adjacent symbols to cancel the known interference without channel estimation. To do so, we obtain a new signal $t(k)$ by combining $y(k)$ and $y(k+1)$ as follows:

$$
\begin{gathered}
t(k)=y(k)-\frac{x_{1}(k)}{x_{1}(k+1)} y(k+1)=\left(x^{\prime}(k)-\frac{x_{1}(k)}{x_{1}(k+1)} x^{\prime}(k+1)\right)+ \\
n(k)-\frac{x_{1}(k)}{x_{1}(k+1)} n(k+1)-x_{1}(k) \Delta(k) \\
\quad k \in\{1,2, \cdots N-1\}
\end{gathered}
$$

In (9), almost all the interference terms have been removed from $t(k)$. However, the signal of interest to us is DSN, $x^{\prime}(k)+n(k)$, rather than $t(k)$. In the next step, we show how to extract DSN from $t(k)$.

\section{Step 2: DSN recovery}

In (9), the target signal $x^{\prime}(k)$ is distorted into the form of $t(k)$ after the interference cancellation step. At first glance, $t(k)$ may appear to be the signal $x^{\prime}(k)$ passing through an Inter-Symbol Interference (ISI) channel, in which case traditional ISI equalization schemes such as filtering, Viterbi detection and Belief Propagation (BP) [13] detection, could be used to recover $x^{\prime}(k)$. However, an important difference is the correlated noise in (9) for adjacent symbols $t(k)$ and $t(k+1)$. Although Viterbi/BP detection achieves optimal MAP performance for independent noise in ISI equalization, its performance is far from optimal for the recovery of the target signal here because of the correlated noise, as will be shown in our numerical simulation.

We proposed two schemes to recover DSN with near optimal performance in [1]. The BKIC-S scheme averages all the $t(k)$ to find an estimate of the DSN. The residual error in $[1$, eq. (8)] is equivalent to the one in (7). The other recover scheme is BKIC-RBP scheme, which performs better than BKIC-S.

The target of BKIC-RBP is to find a vector $\boldsymbol{x}^{\prime}+\boldsymbol{n}$ to maximize

$$
\begin{aligned}
& P\left(\boldsymbol{x}^{\prime}+\boldsymbol{n} \mid \boldsymbol{t}\right) \propto P\left(\boldsymbol{t} \mid \boldsymbol{x}^{\prime}+\boldsymbol{n}\right) P\left(\boldsymbol{x}^{\prime}+\boldsymbol{n}\right) \\
& =\prod_{k} P\left(t(k) \mid x^{\prime}(k)+n(k), x^{\prime}(k+1)+n(k+1)\right) P\left(\boldsymbol{x}^{\prime}+\boldsymbol{n}\right) .
\end{aligned}
$$

Based on (10), a corresponding Tanner Graph can be established as in Fig. 2, where the messages being passed between the variable nodes and the check nodes are the probability density functions of the variable nodes on the left hand side.

An important subtlety in the Tanner graph is that $\boldsymbol{x}{ }^{\prime}+\boldsymbol{n}$ is treated as "signal symbols". With respect to Fig. $2, \boldsymbol{x}{ }^{\prime}+\boldsymbol{n}$ is the target signal that will be fed to the conventional receiver after the interference cancellation process. With reference to
(9), the observation $t(k)$ are made up of adjacent "signal symbols" plus noise in the cancellation process, which is $-x_{1}(k) \Delta(k)$ and does not include $n(k)$ and $n(k+1)$. The noise $n(k)$ will be dealt with by the conventional receiver later.

\section{Parallel BKIC-RBP}

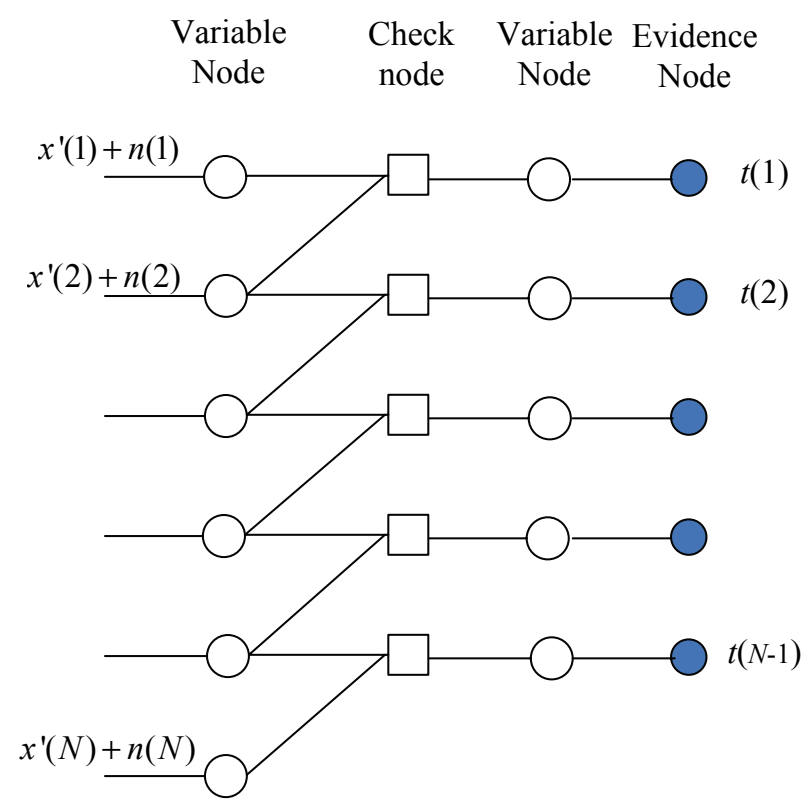

Fig. 2. Tanner Graph for BKIC-RBP, where blank circles are the variable nodes and the filled circles are the evidence nodes and the rectangles are the check nodes

In [1], a successive iteration algorithm was used for the BKIC-RBP scheme. In particular, since the Tanner graph in Fig. 2 does not include any cycle, one iteration is enough to obtain the optimal MAP performance. The iteration consists of two message update processes. One successively updates the messages from top to bottom. The other process successively updates them from bottom to top. Although the total complexity of the successive algorithm is low, it is time consuming because in each process, the update of one message depends on the update of the previous message. Supposing the processing of one message is $\tau$ and the packet length is $N$. The total time of $2(N-1) \tau$ is needed to update the whole packet since there are $2(N-1)$ messages to be successively updated as shown in Fig. 2. In other words, the processing time in successive BKIC-RBP depends on the packet length and may be very large.

Now, we propose a parallel algorithm for BKIC-RBP, which updates messages associated with the edges in Fig. 2 in a parallel way. As a result, the processing time only depends on number of iterations of the algorithm. The detail of the algorithm is as follows.

The parallel BP algorithm includes three critical steps: initializing the messages, updating the messages at the variable nodes and updating the messages at check nodes in an iterative way. 


\section{Message initialization:}

The variable nodes $\boldsymbol{x}^{\boldsymbol{\prime}+\boldsymbol{n}}$ on the left side of Fig. 2 are not associated with any channel outputs, and we initialize the PDF of $\boldsymbol{x}$ ' $\boldsymbol{n}$ with the a priori probabilities. The variable $x$, adopts a discrete value, and the discrete distribution is determined by the constellation set and the channel coefficient. Without assuming specific channel information, the upper bound of the interference power, $P_{\max }$, can be derived easily. Then we assume $x^{\prime}$ is uniformly distributed between $-\sqrt{P_{\max }}$ and $\sqrt{P_{\max }} 1$. Since the noise is of Gaussian distribution, the messages (i.e., the a priori probabilities) associated with the leftmost edges in the Tanner graph can be expressed as

$$
\begin{aligned}
& p_{x^{\prime}+n}(a)=p_{x^{\prime}+n}\left(x^{\prime}+n=a\right)=\int_{-\sqrt{P_{\text {max }}}}^{\sqrt{P_{\text {max }}}} p_{x^{\prime}}\left(x^{\prime}=s\right) p_{n}(n=a-s) d s \\
& =\frac{1}{2 \sqrt{P_{\max }} \sqrt{2 \pi} \sigma} \int_{-\sqrt{P_{\max }}}^{\sqrt{P_{\max }}} \exp \left(-(a-s)^{2} / 2 \sigma^{2}\right) d s \square f(a)
\end{aligned}
$$

For each left variable node $x^{\prime}(k)+n(k)$, there are two connecting edges, whose associated messages are initialized to the one in (11).

\section{Message updates at the check nodes:}

For each check node, we need to calculate the PMF for the left variable nodes connected to it. Consider a check node connected to the right evidence node $t(k)$. The two left variable nodes connected to it are $x^{\prime}(k)+n(k)$ and $x^{\prime}(k+1)+n(k+1)$. Given the PDF of one connected left variable node, $p_{x^{\prime}(k)+n(k)}^{m}(a)$ which is obtained in the $m$-th iteration, then the PDF of the other connected left variable node can be calculated according to (9) as

$$
\begin{aligned}
& p_{x^{\prime}(k+1)+n(k+1)}^{(m+1)}(a) \propto \\
& \int p_{x^{\prime}(k)+n(k)}^{(m)}\left(\frac{x_{1}(k)}{x_{1}(k+1)} a+t(k)+x_{1}(k) s\right) e^{-s^{2} / 2 \sigma_{\Delta}^{2}} d s
\end{aligned}
$$

where $\sigma_{\Delta}^{2}$ is the variance of the interference term $\Delta(k)$, and $p_{x^{\prime}+n}^{(0)}$ is the initial distribution in (11). Analogous to (12), the PDF of $x^{\prime}(k)+n(k)$ can be updated from the PDF of $x^{\prime}(k+1)+n(k+1)$ and the observation $t(k)$ based on the following equation:

$$
p_{x^{\prime}(k)+n(k)}^{(m+1)}(a) \propto \int p_{x^{\prime}(k+1)+n(k+1)}^{(m)}\left(\frac{x_{1}(k+1)}{x_{1}(k)} a-\frac{x_{1}(k+1)}{x_{1}(k)} t(k)-x_{1}(k+1) s\right) e^{-s^{2} / 2 \sigma_{\Delta}^{2}} d s
$$

Message updates at the variable nodes:

We only need to update the messages associated with the left

\footnotetext{
1 A more accurate distribution of $x{ }^{\prime}+n$ should improve the performance of BKIC-RBP. Fortunately, BKIC-RBP with the approximate a prior distribution still performs very well, as demonstrated by our simulation results later.
}

variable nodes $x$ ' $+n$. Each left variable node is connected by three edges. The message associated with the most left edge is the a priori information in (11) and the messages associated with the two edges between the left variable node and the check nodes are denoted by $p_{x^{\prime}(k)+n(k)}^{m}(a)$ and $q_{x^{\prime}(k)+n(k)}^{m}(a)$ after the $m$-th iteration. In the $(m+1)$-th iteration, the message $p_{x^{\prime}(k)+n(k)}^{(m)}(a)$ is updated as

$$
p_{x^{\prime}(k)+n(k)}^{(m+1)}(a)=\frac{1}{T} q_{x^{\prime}(k)+n(k)}^{(m)}(a) \cdot f(a) .
$$

where $T$ is the normalizing factor and $f(a)$ is the a priori PDF of $x^{\prime}+n$ due to the unavailability of the evidence node information. The message $q_{x^{\prime}(k)+n(k)}^{(m)}(a)$ is updated in a similar way

$$
q_{x^{\prime}(k)+n(k)}^{(m+1)}(a)=\frac{1}{T} p_{x^{\prime}(k)+n(k)}^{(m)}(a) \cdot f(a) .
$$

\section{Final Decision:}

After a predefined number of iterations, $M$, the processing stops. Consider a variable node with $x^{\prime}+n$. Suppose the messages associated with the three edges connected to the $k$-th variable node are $p_{x^{\prime}(k+1)+n(k+1)}^{(M)}(a), q_{x^{\prime}(k+1)+n(k+1)}^{(M)}(a)$, and the initial distribution $p_{x^{\prime}(k+1)+n(k+1)}(a)$. Then the final probability distribution of $x^{\prime}+n$ can be calculated as

$$
p_{x^{\prime}(k)+n(k)}^{f}(a)=\frac{1}{T} p_{x^{\prime}(k)+n(k)}^{(M)}(a) \square q_{x^{\prime}(k)+n(k)}^{(M)}(a) \square p_{x^{\prime}(k)+n(k)}(a)
$$

where $T$ is the normalizing factor. $p_{x^{\prime}(k)+n(k)}^{f}(a)$ contains all the information about $x^{\prime}(k)+n(k)$ and it can be fed to the traditional detection block for further target data detection. In order to compare with the traditional KIC scheme, the estimate of $x^{\prime}+n$ is

$$
z(k)=\underset{a}{\arg \max } p_{x^{\prime}(k)+n(k)}^{f}(a)
$$

It can be seen that the number of iterations in the new parallel algorithm does not depend on the packet length any more. Although the processing load in each iteration is higher than that in the successive algorithm, we can still assume the same processing time $\tau$ provided a large number of parallel processing hardware. Then the total time needed is $M \tau$, which does not depend on the packet length. In general, it is much less than the successive algorithm time $2(N-1) \tau$.

\section{Numerical Simulation}

This section presents numerical simulation results for the performance of parallel BKIC-RBP algorithm. We again take node $N_{1}$ as an example for simulation. Without loss of generality, we assume the BPSK modulation, i.e., $x_{1}, x_{2} \in\{1,-1\}$ and $P_{1}=P_{2}$. As is clear from the earlier 
discussion, the form of $x_{2}$ does not affect the interfernece cancellation operation. Thus, without loss of generality in BKIC, we simply assume flat fading with unit channel coefficient for $h_{2}^{2}$.

For channel $h_{1}$, continuous flat fading is simulated. In particular, we set the interference channel to single path continuous fading with the first order Markov channel mode in as in [10] and the parameter $\alpha$ is set to $1-10^{-3}$, which corresponds to a fast fading channel. In our simulation, $\sigma_{\Delta}^{2}$ is set to 0.001 . The noise variance is set to unit and the power of the signals varies in the simulation. The packet length $N$ is set to 128 .

For both parallel and successive BKIC-RBP, we quantize the messages (the PDFs) into discrete form to enable simulation with Matlab. And the quantization interval is 0.025 for $1-7 \mathrm{~dB}$ and it is 0.0125 for $8-10 \mathrm{~dB}$. The number of iteration $M$ is set to 30 for the parallel BKIC-RBP.

For comparison purposes, we also simulate the scheme of Noise Predictive Belief Propagation (NPBP) in [14] for the second step of BKIC. In our simulation, the length of the whitening filter in [14] is set to two. NPBP is also a parallel processing algorithm. The complexity of BKIC-NPBP is $30(4 N-6)$ message update operations plus 3 whitening operations, which is much higher than successive BKIC-RBP and parallel BKIC-RBP.

Fig. 3, and Fig. 4 show the residual interference variance and BER of the BKIC schemes. For comparison, we also give the BER performance of the traditional known interference cancellation (traditional KIC) scheme, which is in fact the BKIC-S scheme in [1]. From both figures, BKIC-RBP outperforms all the other schemes by at least $1 \mathrm{~dB}$.

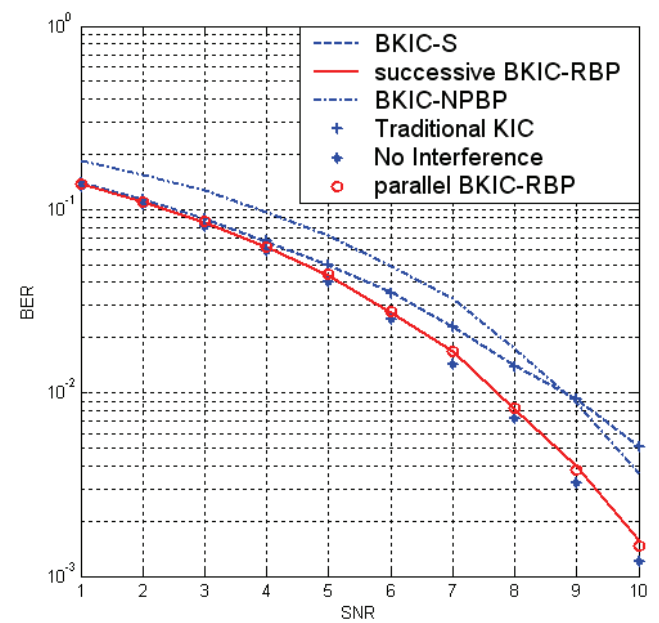

Fig. 3. BER performance of different BKIC schemes

\footnotetext{
${ }^{2}$ note: with respect to Fig. 2 , it is in the conventional receiver that the channel characteristics of the target signal that matters, and it is over there that the actual fading characteristics of the target signal channel come into play
}

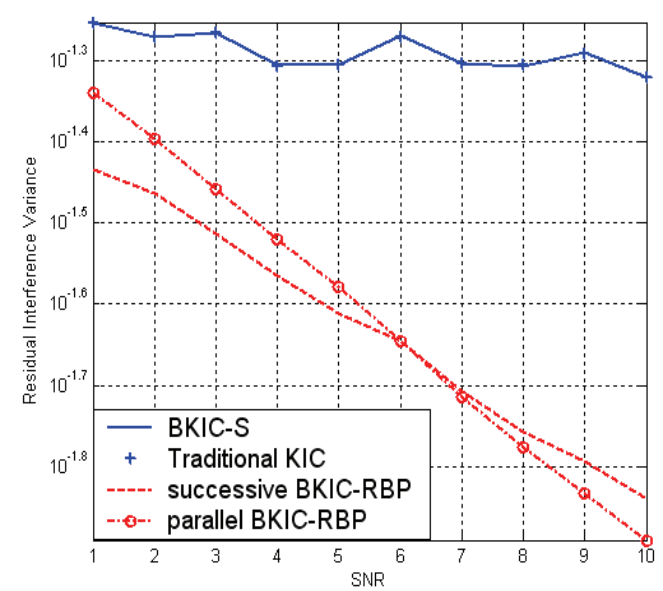

Fig. 4. Residual interference of difference BKIC schemes

\section{Conclusions}

Although there has been much theoretical work in known interference cancellation, deployments of the previously proposed schemes are difficult because of their needs for accurate channel estimation and their high complexity. To our knowledge, the first effective blind known interferencecancellation scheme was proposed in [1], which does not require estimation of the interference channel.

The BKIC-RBP scheme in [1] can achieve the optimal MAP performance, which is much better than the traditional KIC scheme. However, the successive message updating requires much processing time. In order to reduce the processing time, a parallel BKIC-RBP scheme is proposed in this paper. The processing time of the new scheme only depends on the iteration number rather than the packet length as the successive BKIC-RBP scheme in [1]. At the same time, the good cancellation performance is maintained.

\section{Acknowledgement:}

This work is partially supported by AoE grant E-02/08 and the General Research Funds Project No. 414911. This work is also partially supported by the China 973 Program (Project No. 2012CB315904), by the National Science Foundation of China (Grant No. 60902016), the NSF of Guangdong (Grant No. 10151806001000003) and the NSF of Shenzhen (Grant No. JC201005250034A).

\section{References:}

[1] S. Zhang, S. Liew and H. Wang, "Blind known interference cancellation", to Appear in IEEE JSAC 2012.

[2] C. Qin, N. Santhapuri, S. Sen, S. Nelakuditi ” Known Interference Cancellation: Resolving Collisions due to Repeated Transmissions" in Proc. Fifth IEEE Workshop on Wireless Mesh Networks (WIMESH 2010), June 2010

[3] S. Zhang, S. Liew, and L. Lu "Physical layer network coding schemes over finite and infinite fields", Proc. IEEE Globecom 2008, Dec. 2008.

[4] S. Katti, S. Gollakota, and D. Katabi, "Embracing Wireless Interference: Analog Network Coding”. Proc. ACM SIGCOMM, 2007.

[5] L. Lu, T. Wang, S. C. Liew, and S. Zhang, "Implementation of physical-layer network coding," submitted to Physical Communication, 
available at http://arxiv.org/abs/1105.3416.

[6] F. Gao, R. Zhang, and Y-C Liang, "On Channel Estimation for Amplify-and-Forward Two-Way Relay Networks" IEEE Transactions on Communications, Vol. 57, Issue 10, October 2009

[7] L. Song, Y. Li, A.Huang, B. Jiao, and A. V. Vasilakos, "Differential modulation for bidirectional relaying with analog network coding," IEEE Transactions on Signal Processing, vol. 58, no. 7, pp. 3933-3938, 2010.

[8] T. Cui, F. Gao, and C. Tellambura, "Differential modulation for two-way wireless communications: a perspective of differential network coding at the physical layer," IEEE Transactions on Communications, vol. 57, no. 10, pp. 2977-2987, 2009.

[9] Q. Sun, D. C. Cox, and H. C. Huang, "Estimation of continuous flat fading MIMO channels," IEEE Trans. Wireless Commun., vol. 1, pp. 549-553, Oct. 2002.

[10] Y. Zhu, D. Guo and M. Honig, "A message-passing approach for joint channel estimation, interference mitigation, and decoding " IEEE Transactions on Wireless Communications, Vol 8(12), pp. $6008-6018$, Dec. 2009.

[11] W. C. Jakes Jr., Microwave Mobile Communications. New York: Wiley, 1974.

[12] J. G. Proakis, Digital Communications. New York: McGraw Hill, 1995.

[13] M. N. Kaynak, T. M. Duman, and E. M. Kurtas, "Belief propagation over frequency selective fading channels," Proc. IEEE VTC'04-Fall, Sept. 2004, vol. 2, pp. 1367 - 1371.

[14] M. N. Kaynak, T. M. Duman, and E. M. Kurtas, "Noise predictive belief propagation," IEEE Transactions on Magnetics, vol. 41, no. 12, pp. 4427-4434, 2005.

[15] S. Zhang, S. Liew and P. Lam, "Physical layer network coding", in Proc. ACM Mobicom 2006.

[16] A. Papadogiannis, A-G. Burr and M. Tao, "On the maximum achievable sum-rate of interfering two-way relay channels," IEEE Comm. Letters, vol. 16, no. 1, pp. 72-75, Jan. 2012. 